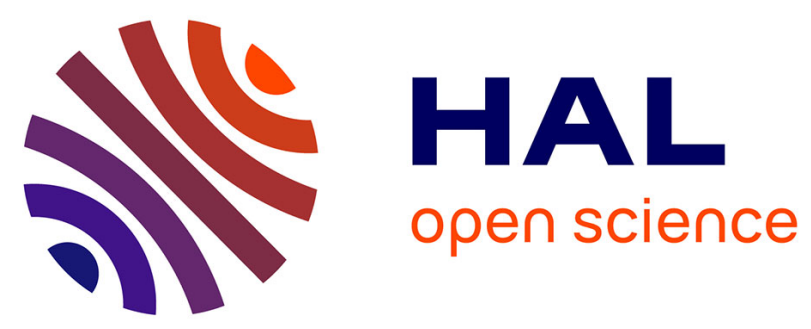

\title{
On oriented labelling parameters
}

Daniel Gonçalves, André Raspaud, M.A. Shalu

\section{To cite this version:}

Daniel Gonçalves, André Raspaud, M.A. Shalu. On oriented labelling parameters. Formal Models, Languages and Applications, 66, pp.33-45, 2006, 978-981-277-303-6. 10.1142/9789812773036_0003 . hal-00353655

\section{HAL Id: hal-00353655 \\ https://hal.science/hal-00353655}

Submitted on 22 Jan 2022

HAL is a multi-disciplinary open access archive for the deposit and dissemination of scientific research documents, whether they are published or not. The documents may come from teaching and research institutions in France or abroad, or from public or private research centers.
L'archive ouverte pluridisciplinaire HAL, est destinée au dépôt et à la diffusion de documents scientifiques de niveau recherche, publiés ou non, émanant des établissements d'enseignement et de recherche français ou étrangers, des laboratoires publics ou privés.

\section{다)(1) $(5$}

Distributed under a Creative Commons Attribution - NonCommercial| 4.0 International 


\title{
ON ORIENTED LABELLING PARAMETERS
}

\author{
Daniel Gonçalves, André Raspaud and M. A. Shalu* \\ LaBRI UMR CNRS 5800, Université Bordeaux I, \\ 33405 Talence Cedex, France \\ E-mail: raspaud@labri.fr
}

\begin{abstract}
We introduce two notions, (i) $L(p, q)$ labelling for oriented graphs and (ii) oriented $L(p, q)$ labelling, to explore frequency assignment problem under half-duplex setting and compute bounds of oriented labelling of special classes of graphs: trees, bipartite graphs and planar graphs. Moreover we prove that the span of the $L(p, 1)$ labelling for oriented graphs is bounded by $\left\lfloor\frac{\Delta^{2}}{2}\right\rfloor+p \Delta$.
\end{abstract}

\section{Introduction}

The frequency assignment problem (FAP) arises in wireless communication systems. There are several models based on genetic algorithms, neural networks, constrained programming and combinatorial enumeration to explore and optimize different features of FAP such as available frequencies and limiting interference among radio signals. In 1970, Metzger ${ }^{15}$ introduced graph coloring techniques as a tool to optimize the frequency spectrum used in FAP. Motivated by FAP, Hale ${ }^{11}$ developed the concept of $T$-coloring and it lead to many indepth graph theoretical results. A common feature of all these graph theoretical models of FAP is that they assume communication is viable in both direction (duplex) between two radio trasmitters and hence model FAP as a non-oriented graph. This is far away from reality. In fact, Aardal et al emphasised the importance of "direction/orientation" of transmission in their recent survey (for details see Ref. 1 page 4) on FAP.

*This work has been done during a visit of the third author at LaBRI. This visit was sponsored by the french ministry of education and research. 
In this paper, we explore FAP under half-duplex setting (i.e., there are radio transimitters in a network in which at most one way transimission is effective between any two of them). Hence FAP can be modeled as an oriented multi graph. As a preliminary step, we focus on oriented simple graphs.

\section{Oriented Vertex Partitioning Problems}

The partitioning of the vertex set of a non-oriented graph into minimum number of subsets in which each subset possesses a special property is a fundamental graph theory problem with many applications. For example: vertex coloring, star (vertex) coloring and clique cover problem. We suggest a general frame work to incorporate "orientation" into various vertex partitioning problems. Two subsets $A, B$ of the vertex set of an oriented graph is called one way oriented if all edges with one end vertex in $A$ and other in $B$ are oriented from $A$ (respectively $B$ ) to $B$ (respectively $A$ ).

Oriented vertex partitioning problem: Partition the vertex set of an oriented graph into minimum number of pairwise one way oriented subsets in which each set possesses a special property.

Oriented coloring is a well known oriented vertex partitioning problem. ${ }^{17,18}$

\section{Notation and Terminology}

In this paper, we consider only finite (oriented and non-oriented) simple graphs. As usual, $N^{+}(v)=\{u: \overrightarrow{v u} \in E(\vec{G})\}$ and $N^{-}(v)=\{u: \overrightarrow{u v} \in$ $E(\vec{G})\}$. A proper $k$-coloring of the vertices of a non-oriented graph $G$ is an assignement $f$ of integers (or labels), using at most $k$ colors (or labels), to the vertices of $G$ such that $f(u) \neq f(v)$ if the vertices $u$ en $v$ are adjacent in $G$. The chromatic number of a graph $G$ is the smallest integer $k$ such that $G$ has a proper $k$-coloring. A proper $k$-coloring of the vertices of a nonoriented graph is called acyclic if the subgraph induced by the vertices of any two color classes has no cycle. The acyclic chromatic number of a graph $G$ is the smallest integer $k$ such that $G$ has an acyclic $k$-coloring. We will say that a graph $G$ is $k$-acyclic if its acyclic chromatic number is at most $k$. The length of the shortest path joining two vertices $x, y$ in a non-oriented graph is called distance between $x$ and $y$. A path with an orientation such that all internal vertices have both in-degree and out-degree one is called an oriented path. The length of a shortest oriented path joining two vertices $x$, $y$ in an oriented graph is called oriented distance between $x$ and $y$. The girth 
of a non-oriented graph $G$ is the length of its smallest cycle. Let $\vec{G}=(V, A)$ and $\vec{H}=(U, B)$ be two oriented graphs. A homomorphism from $\vec{G}$ to $\vec{H}$ is a map $f: V \longrightarrow U$ which preserves adjacency, that is for any arc $\overrightarrow{x y} \in A$, the corresponding arc $f(x) f(y) \in B$.

Oriented coloring ${ }^{17,18}$ : Let $\vec{G}=(V, A)$ be an oriented graph. A map $c: V \longrightarrow\{1,2, \ldots, k\}$ is called a $k$-oriented coloring of $\vec{G}$ if it satisfies the following conditions:

(i) For any edge of $\vec{G}, \overrightarrow{x y}, c(x) \neq c(y)$.

(ii) There are no two edges of $\vec{G}, \overrightarrow{x y}, \overrightarrow{u v}$ such that $c(x)=c(v)$ and $c(y)=$ $c(u)$.

The least integer $k$ in which $\vec{G}$ has $k$-oriented coloring is called the oriented chromatic number $\vec{\chi}(\vec{G})$ of $\vec{G}$. The oriented chromatic number $\vec{\chi}(G)$ of a non-oriented graph $G$ is defined as $\max \{\vec{\chi}(\vec{G}): \vec{G}$ is an orientation of $G\}$.

Note that the condition (i) of oriented coloring ensures that adjacent vertices do not belong to same color class. The condition (ii) guarantees that any two color classes preserve one way oriented property in an oriented graph. Moreover, $\chi(G) \leq \vec{\chi}(G)$ where $\chi(G)$ denotes the chromatic number of $G$. Since $\vec{\chi}\left(K_{n, n}\right)=2 n, \vec{\chi}(G)$ has no upper bound as a function of $\chi(G)$. Raspaud and Sopena ${ }^{18}$ proved that the oriented chromatic number of a planar graph is at most 80 .

A suggestion of Roberts to distinguish close and very close transmitters in a wireless communication system led Griggs and $\mathrm{Yeh}^{10}$ to propose a variation of FAP as labelling the vertices of a non-oriented graph with a condition at a distance two (known as $L(2,1)$-labelling). Georges and Mauro $^{8}$ generalized this as follows:

$L(p, q)$-Labelling: Let $G=(V, E)$ be a non-oriented graph and $p, q$ be two positive integers. A map $L: V \longrightarrow\{0,1, \ldots, k\}$ is called a $k-L(p, q)$ labelling if it satisfies the following.

(i) For any edge $x y \in E,|L(x)-L(y)| \geq p$.

(ii) For any pair of vertices $x, y$ at a distance $2,|L(x)-L(y)| \geq q$.

The span, $\lambda_{p, q}(G)$, of $G$ is defined as $\min \{k: G$ has a $k-L(p, q)$ labelling . For convenience, we prefer $\lambda_{p}(G)$ to $\lambda_{p, 1}(G)$.

We cite a few known results in $L(p, q)$-labelling problems.

(1) $L(2,1)$-labelling problem is $N P$-complete. ${ }^{10}$

(2) For a tree $T$ with maximum degree $\Delta, \Delta+1 \leq \lambda_{2}(T) \leq \Delta+2 .{ }^{10}$ 
(3) For a graph $G$ with maximum degree $\Delta, \lambda_{p}(G) \leq \Delta^{2}+(p-1) \Delta-2 .{ }^{9}$

\section{Two Oriented Variations of $L(p, q)$-Labelling}

In this section, we extend $L(p, q)$-labelling to oriented graphs and propose a new oriented vertex partitioning problem.

$L(p, q)$-Labelling for oriented graphs: Let $\vec{G}=(V, A)$ be an oriented graph and $p, q$ be two positive integers. A map $L: V \rightarrow\{0,1, \ldots, k\}$ is called a $k-L(p, q)$-labelling of $\vec{G}$ if it satisfies the following.

(i) For any edge $\overrightarrow{x y} \in A,|L(x)-L(y)| \geq p$.

(ii) For any pair of vertices $x, y$ at an oriented distance $2,|L(x)-L(y)| \geq q$.

The span, $\lambda_{p, q}^{o}(\vec{G})$, of $\vec{G}$ is defined as $\min \{k: \vec{G}$ has a $k-L(p, q)$ labelling $\}$. The span of a non-oriented graph $G, \lambda_{p, q}^{o}(G)$, is defined as max $\left\{\lambda_{p, q}^{o}(\vec{G}): \vec{G}\right.$ is an orientation of $\left.G\right\}$. For convenience (when $q=1$ ), we denote $\lambda_{p, 1}^{o}(G)=\lambda_{p}^{o}(G)$.

This notion is already studied by Chang et al. ${ }^{6}$ They give interesting bounds for the span of oriented graphs with a specified longest dipath length.

Oriented $L(p, q)$-Labelling: Let $\vec{G}=(V, A)$ be an oriented graph and $p$ be a positive integer. A map $l: V \rightarrow\{0,1, \ldots, k\}$ is called a $k$-oriented $L(p, q)$-labelling if it satisfies the following.

(i) For any edge $\overrightarrow{x y} \in A,|l(x)-l(y)| \geq p$.

(ii) For any pair of vertices $x, y$ at an oriented distance $2,|l(x)-l(y)| \geq q$.

(iii) There are no two edges $\overrightarrow{x y}, \overrightarrow{u v}$ such that $l(x)=l(v)$ and $l(y)=l(u)$.

The span, $\vec{\lambda}_{p, q}(\vec{G})$, of $\vec{G}$ is defined as $\min \{k: \vec{G}$ has a $k$-oriented $L(p, q)$ labelling $\}$. The span of a non-oriented graph $G, \vec{\lambda}_{p, q}(G)$, is defined as max $\left\{\vec{\lambda}_{p, q}(\vec{G}): \vec{G}\right.$ is an orientation of $\left.G\right\}$. For convenience (when $q=1$ ), we denote $\vec{\lambda}_{p, 1}(G)=\vec{\lambda}_{p}(G)$.

Remarks: There is a distinction between the $L(p, q)$-labelling of oriented graphs and the oriented $L(p, q)$-labelling. Note that any two oriented $L(p, q)$-labelling color classes (i.e. set of vertices with same label) are one way oriented. But $L(p, q)$-labelling doesn't guarantee one way orientedness of its color classes. A pair of color classes in a $L(p, q)$-labelling can be viewed as a union of two (disconnected) pairs of one way oriented sets (see Fig. 1). Hence, an oriented $L(p, q)$-labelling of an oriented graph $\vec{G}$ is also a $L(p, q)$ labelling of $\vec{G}$. The graph $\vec{H}$ in Fig. 1, with $\lambda_{1}^{o}(\vec{H})=3$ and $\vec{\lambda}_{1}(\vec{H})=4$, shows that the converse is not true. 


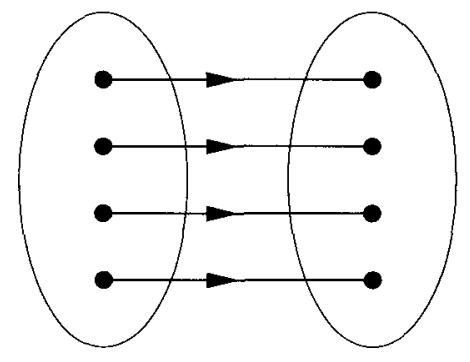

Two color classes in an oriented $\mathrm{L}(\mathrm{p}, \mathrm{q})$-labelling

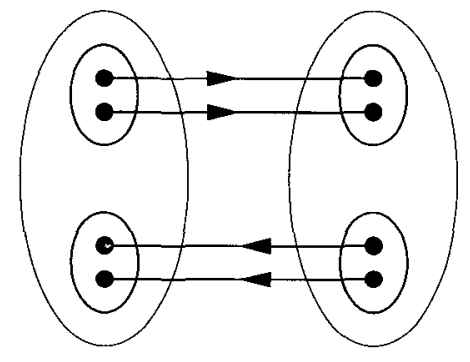

Two color classes in an $\mathrm{L}(\mathrm{p}, \mathrm{q})$-labelling

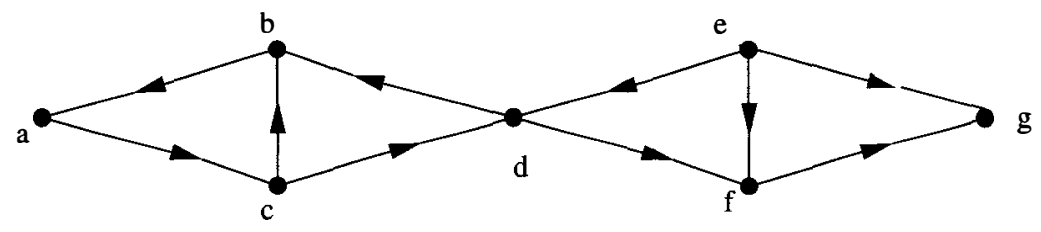

Fig. 1. Oriented color classes and the graph $\vec{H}$.

Let $H$ be a subgraph of $G$. Then $\overrightarrow{\lambda_{p}}(H) \leq \overrightarrow{\lambda_{p}}(G)$. Oriented $L(p, q)$ labelling is a generalization of oriented coloring. In particular, $\vec{\lambda}_{1}(\vec{G})=$ $\vec{\chi}(\vec{G})-1$ (we do allow '0' as a label). Let $I_{0}, I_{1}, \ldots, I_{\vec{\chi}-1}$ be a set of oriented color classes of $\vec{G}$. We produce a $(\vec{\chi}(\vec{G})-1) p$-oriented $L(p, p)$-labelling of $\vec{G}$ by assigning the label $j p$ to each vertex in the set $I_{j}$ for $0 \leq j \leq \vec{\chi}(\vec{G})-1$. It is easy to see that if there is a homomophism $h: \vec{G} \rightarrow \vec{H}$, then $\vec{\lambda}_{p, q}(\vec{G}) \leq$ $\vec{\lambda}_{p, q}(\vec{H})$. Indeed, given a $k$-oriented $L(p, q)$-labelling $l_{H}$ of $\vec{H}$, we define a $k$-oriented $L(p, q)$-labelling $l_{G}$ of $\vec{G}$, by $l_{G}(v)=l_{H}(h(v))$, for all $v \in V_{G}$. We also note that a $L(p, q)$-labelling of a non-oriented graph $G$ is a $L(p, q)$ labelling of any orientation of $G$. By definition, an oriented $L(p, q)$-labelling of an oriented graph is also its $L(p, 1)$-labelling. These remarks prove the following lemma.

Lemma 1: Let $G$ be a non-oriented graph. Then

(i) For $p \geq q>0, \vec{\chi}(G)-1 \leq \vec{\lambda}_{p, q}(G) \leq(\vec{\chi}(G)-1) p$.

(ii) $\lambda_{p, q}^{o}(G) \leq \lambda_{p, q}(G)$.

(iii) $\lambda_{p, q}^{o}(G) \leq \vec{\lambda}_{p, q}(G)$. 
There is no trivial relation between $\lambda_{p, q}$ and $\vec{\lambda}_{p, q}$. Indeed, the graphs $H_{1}$ and $H_{2}$ depicted in Fig. 2 are such that $\lambda_{p, q}\left(H_{1}\right)<\vec{\lambda}_{p, q}\left(H_{1}\right)$ and $\lambda_{p, q}\left(H_{2}\right)>$ $\vec{\lambda}_{p, q}\left(H_{2}\right)$.
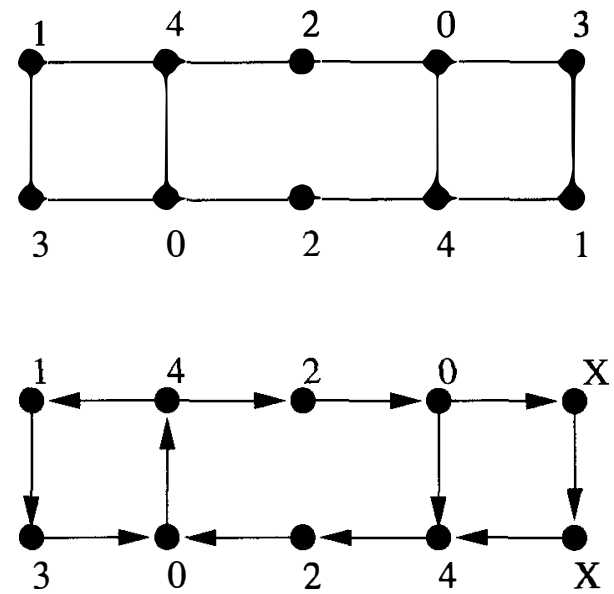

$\mathrm{H}_{1}$
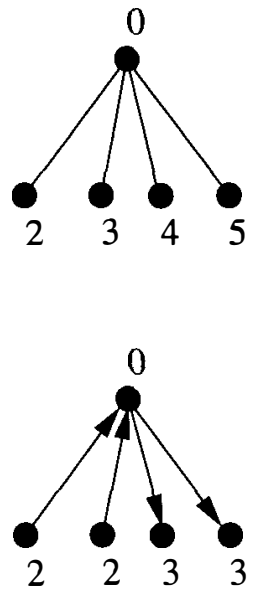

$\mathrm{H}_{2}$

Fig. 2. The graphs $H_{1}$ and $H_{2}$.

For a compelete graph $K_{n}, \overrightarrow{\lambda_{p}}\left(K_{n}\right)=\left(\vec{\chi}\left(K_{n}\right)-1\right) p$. Moreover, $\overrightarrow{\lambda_{2}}\left(C_{5}\right)=$ $4=\vec{\chi}\left(C_{5}\right)-1$. Though the bounds in the Lemma 1 (i) is tight for certain graphs, we could significantly improve this result for the class of trees.

\section{Oriented $L(p, q)$-Labelling for Trees}

A star, $S$, is a tree with a special vertex $x$ and all other vertices of $S$ are adjacent to $x$. A double star, $D$, is a tree with a special pair of adjacent vertices $x, y$ and all other vertices of $D$ are adjacent to either $x$ or $y$ (see Fig. 3).

We denote $P_{k}$ the paths with $k$ vertices. Any tree $T \neq K_{1}, K_{2}$ has a $P_{3}$ as a subgraph. A tree $T\left(\neq K_{1}, K_{2}\right)$ with no $P_{5}$ as a subgraph is either a star or a double star. The minimal span for the oriented $L(p, q)$-labelling of an unoriented tree $T$ is easily computable with the following theorem. 


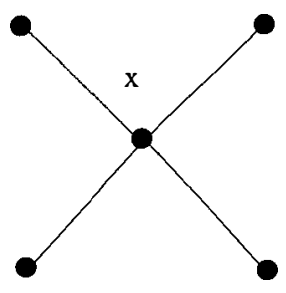

Star

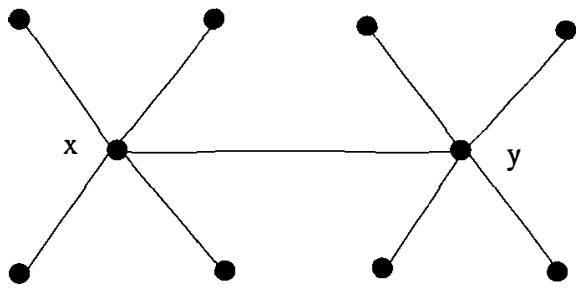

Double star

Fig. 3. Star and Double Star.

Theorem 1: Let $T$ be an unoriented tree. Then

$$
\vec{\lambda}_{p, q}(T)= \begin{cases}0 & \text { if } T=K_{1} \\ p & \text { if } T=K_{2} \\ p+q & \text { if } T \text { is a star or a double star } \\ p+2 q & \text { else (i.e. } P_{5} \text { is a subgraph of } T \text { ) }\end{cases}
$$

The two first cases are trivial. We begin by proving that in the two last cases the span cannot be decreased. Since $\vec{\lambda}_{p, q}(H) \leq \vec{\lambda}_{p, q}(G)$ if $H$ is a subgraph of $G$, we just have to note that $\vec{\lambda}_{p, q}\left(P_{3}\right)=p+q$ and $\vec{\lambda}_{p, q}\left(P_{5}\right)=$ $p+2 q$. Now we show how to label the trees.

Case 1: Let $T$ be a star with a special vertex $x$. We construct an oriented $L(p, q)$-labelling of $\vec{T}$ by assigning 0 to the special vertex $x, p$ to all vertices of $N_{\vec{H}}^{+}(x)$, and $p+q$ to all vertices of $N_{\vec{H}}^{-}(x)$.

Case 2: Let $T$ be a double star with special vertices $x, y$. Without loss of generality, assume that $\overrightarrow{x y} \in E(\vec{H})$. We construct an oriented $L(p, q)$ labelling of $\vec{H}$ by assigning 0 to all vertices of $N_{\vec{H}}^{-}(y), q$ to all vertices of $N_{\vec{H}}^{+}(y), p$ to all vertices of $N_{\vec{H}}^{-}(x)$, and $p+q$ to all vertices of $N_{\vec{H}}^{+}(x)$.

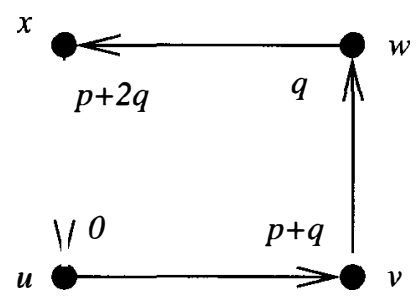

Fig. 4. The graph $\vec{C}_{4}$. 
Case 3: Let $\vec{T}$ be an orientation of $T$. To prove the upperbound, we construct a homomorphism $f: \vec{T} \longrightarrow \vec{C}_{4}$. In Fig. $4, u, v, w$, and $w$ are vertices of $\vec{C}_{4}$ and $0, p+q, q, p+2 q$ are their respective labels in a $p+2 q$ oriented $L(p, q)$-labelling of $\vec{C}_{4}$. First, we pick an arbitaray vertex $a$ of $\vec{T}$ and map $a$ to $u$ (i.e., $f(a)=u$ ). Then, we map the vertices of $N_{\vec{T}}^{+}(a)$ (respectively $N_{\vec{T}}^{-}(a)$ ) into $N_{\vec{C}_{4}}^{+}(u)=\{v\}$ (respectively $\left.N_{\vec{C}_{4}}^{-}(u)=\{x\}\right)$. We continue this process until all vertices of $\vec{T}$ are mapped into $V\left(\vec{C}_{4}\right)$.

\section{Oriented $L(p, 1)$-Labelling of Bipartite Graphs}

Lemma 2: For a complete bipartite graph $K_{m, n}(m, n \geq 1), \overrightarrow{\lambda_{p}}\left(K_{m, n}\right) \leq$ $m+n+p-2$.

Proof: Let $V\left(K_{m, n}\right)=A \cup B$ where $A=\left\{u_{1}, u_{2}, \ldots, u_{m}\right\}$ and $B=$ $\left\{v_{1}, v_{2}, \ldots, v_{n}\right\}$. We construct an oriented $(m+n+p-2)$-labelling of an arbitrary orientation of $K_{m, n}$ by a function $l: V\left(K_{m, n}\right) \longrightarrow\{0,1, \ldots, m+$ $n+p-2\}$ defined as $l\left(u_{i}\right)=i-1$ for $1 \leq i \leq m$ and $l\left(v_{j}\right)=m+j+p-2$ for $1 \leq j \leq n$. Hence $\overrightarrow{\lambda_{p}}\left(K_{m, n}\right) \leq m+n+p-2$.

It is not hard to show that the upper bound in the Lemma is tight if $m=n$, i.e., $\overrightarrow{\lambda_{p}}\left(K_{n, n}\right)=2 n+p-2$. Note that every bipartite graph is a subgraph of a complete bipartite graph. Hence, for a bipartite graph $G$, $\overrightarrow{\lambda_{p}}(G) \leq|V(G)|+p-2$.

\section{Oriented $L(p, 1)$-Labelling and the Acyclic Chromatic Number}

In this section, we supply an upperbound of $\vec{\lambda}_{p}$ of planar graphs based on a method developed by Alon, Marshall, Nesetril, Raspaud and Sopena. ${ }^{2,16,18}$ In fact, they found a homorphism from any oriented $k$-acyclic graph to a special graph, $\vec{M}_{k}$.

Special graph $\vec{M}_{k}$ : Let $\vec{M}_{k}$ be an oriented graph with vertex set $V\left(M_{k}\right)=$ $\left\{\left(i, a_{1}, a_{2}, \ldots, a_{i-1}, a_{i+1}, \ldots, a_{k}\right): 1 \leq i \leq k\right.$ and $\left.a_{j} \in\{0,1\}\right\}$. The edge set of $\vec{M}_{k}$ is defined as follows. Let $x=\left(i, a_{1}, a_{2}, \ldots, a_{i-1}, a_{i+1}, \ldots, a_{k}\right)$ and $y=\left(l, b_{1}, b_{2}, \ldots, b_{l-1}, b_{l+1}, \ldots, b_{k}\right), 1 \leq i<l \leq k$, be two vertices. Then (i) $\overrightarrow{x y} \in E\left(\vec{M}_{k}\right)$ if $\overrightarrow{a_{l} \vec{b}_{i}} \in E(\vec{T})$ and (ii) $\overrightarrow{y x} \in E\left(\vec{M}_{k}\right)$ if $\overrightarrow{b_{i} a_{l}} \in E(\vec{T})$ (see Fig. 5 for $\vec{T})$.

Theorem 2: ${ }^{16}$ Let $\vec{G}$ be an orientation of a $k$-acyclic graph $G$. Then there exists a homomorphism from $\vec{G}$ to $\vec{M}_{k}$. 


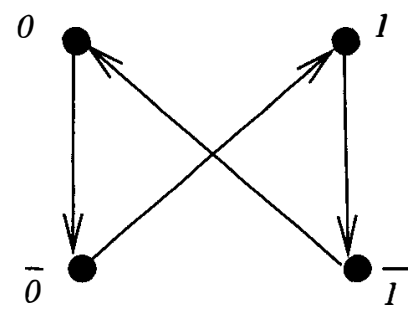

Fig. 5. The graph $\vec{T}$.

Lemma 3: $\vec{\lambda}_{p}\left(\vec{M}_{k}\right) \leq k\left(2^{k-1}-1\right)+p(k-1)$.

Proof: Let $V\left(\vec{M}_{k}\right)=\cup_{i=1}^{k} V_{i}$, where $V_{i}=\left\{\left(i, a_{1}, a_{2}, \ldots, a_{i-1}, a_{i+1}, \ldots\right.\right.$, $\left.\left.a_{k}\right): a_{j} \in\{0,1\}\right\}$. Note that $\left|V_{i}\right|=2^{k-1}$. Then we re-label the vertices of $V_{i}$ as $\left\{v_{i, j}: 1 \leq j \leq 2^{k-1}\right\}, 1 \leq i \leq k$. Now, it is easy to see that the map $f: V\left(\vec{M}_{k}\right) \longrightarrow\left\{0,1, \ldots, k 2^{k-1}+(k-1)(p-1)-1\right\}$ such that $f\left(v_{i, j}\right)=$ $(i-1)\left(2^{k-1}+p-1\right)+j-1,1 \leq i \leq k$ and $1 \leq j \leq 2^{k-1}$, is an oriented $L(p, 1)$ labelling of $\vec{M}_{k}$. Indeed, $f$ is an oriented labelling (coloring), it follows that vertices at distance 2 have different colors, moreover if $u$ and $v$ are adjacent vertices we have $|f(u)-f(v)| \geq p$. Hence $\vec{\lambda}_{p}\left(\vec{M}_{k}\right) \leq k\left(2^{k-1}-1\right)+p(k-1 \phi$

Theorem 3: Let $G$ be a $k$-acyclic graph. Then $\vec{\lambda}_{p}(G) \leq k\left(2^{k-1}-1\right)+$ $p(k-1)$.

Proof: Let $\vec{G}$ be an orientation of $G$. By Theorem 2, there exists a homomorphism from $\vec{G}$ to $\vec{M}_{k}$. Then $\vec{\lambda}_{p}(\vec{G}) \leq \vec{\lambda}_{p}\left(\vec{M}_{k}\right)$. Since $\vec{G}$ is an arbitrary orientation of $G, \vec{\lambda}_{p}(G) \leq \vec{\lambda}_{p}\left(\vec{M}_{k}\right)$. By Lemma $3, \vec{\lambda}_{p}(G) \leq k\left(2^{k-1}-1\right)+p(k-1)$.

A well-known result of Borodin ${ }^{3}$ states that any planar graph is 5-acyclic colorable. In Ref. 5, the authors proved that planar graphs with girth at least 5 (resp. 7) are 4-acyclic colorable (resp. 3-acyclic colorable). Moreover it is well known that graphs with treewidth $k$ are $(k+1)$-acyclic colorable. So we have the following corollary.

Corollary 1: If $G$ is a planar graph, then $\vec{\lambda}_{p}(G) \leq 75+4 p$.

If $G$ is a planar graph with girth at least 5 , then $\vec{\lambda}_{p}(G) \leq 28+3 p$.

If $G$ is a planar graph with girth at least 7 , then $\vec{\lambda}_{p}(G) \leq 9+2 p$.

If $G$ is a graph with treewidth $k$, then $\vec{\lambda}_{p}(G) \leq(k+1)\left(2^{k}-1\right)+p k$. 


\section{8. $L(p, q)$-Labelling of Oriented Graphs}

In Ref. 10, the authors conjectured that for an unoriented graph $G$, $\lambda_{2,1}(G) \leq \Delta^{2}$, where $\Delta$ is the maximum degree of $G$. Much work ${ }^{7,14,9}$ have been done on bounding $\lambda_{p, q}$ by a function of $\Delta$. Here, we prove a similar result for oriented graphs.

Theorem 4: For every directed graph $G=(V, A)$ with maximal degree $\Delta, \lambda_{p, 1}^{o}(G) \leq\left\lfloor\frac{\Delta^{2}}{2}\right\rfloor+p \Delta$.

In a directed graph $G=(V, A), u$ is a 2-neighbor of $v$ if there is a directed 2-path between $u$ and $v$. Given a directed graph $G=(V, A)$, its 2-paths graph $G^{2}=(V, E)$ is an unoritented graph with the same vertex set. There is an edge $u v$ in this graph if and only if there is a directed 2-path in $G$ linking $u$ and $v$. The next lemma gives an interesting property of these graphs.

Lemma 4: For every directed graph $G=(V, A)$ with maximal degree $\Delta$, its 2-paths graph $G^{2}=(V, E)$ is $\left\lfloor\frac{\Delta^{2}}{2}\right\rfloor$-degenerate.

Proof: We prove that for any $S \subseteq V$, the induced graph $G^{2}[S]$ has minimal degree at most $\left\lfloor\frac{\Delta^{2}}{2}-\right\rfloor$. We do so, using a discharging method.

Let the initial charge $\gamma(v)$ of the vertices be $\frac{\Delta^{2}}{2}$ if $v \in S$, or 0 if $v \notin S$. The total charge of the graph is $\frac{\Delta^{2}}{2}|S|$. Then we proceed to the following discharging step, every vertex of $S$ gives the charge $\frac{\Delta}{2}$ to each of its neighbors. We denote $\gamma^{*}$ the new charge of the vertices of $G$. Note that a vertex $v$ with $k$ neighbors in $S$ has charge $\gamma^{*}(v)$ at least $\frac{k \Delta}{2}$.

Now consider the number of oriented 2-paths going through a vertex $v \in V(G)$ and linking two vertices in $S$. Let us denote this number $\pi_{S}(v)$. Note that for a vertex $v$ with $k$ neighbors in $S$, we have that $\pi_{S}(v) \leq$ $\max \{i \times j, i+j=k\}=\left\lfloor\frac{k^{2}}{4}\right\rfloor$, where $i$ and $j$ are respectively the number of incoming and outgoing arcs.

Since $k \leq \Delta$ we have that $\pi_{S}(v) \leq\left\lfloor\frac{k^{2}}{4} \mid \leq \frac{k \Delta}{4} . \leq \frac{\gamma^{*}\left(\frac{v}{2}\right)}{2}\right.$. This implies that the number of edges in $G^{2}[S], \sum_{v \in V(G)} \pi_{S}(v)$, is at most $\frac{1}{2} \sum_{v \in V(G)} \gamma^{*}(v)$. Since the discharging does not change the total charge of the graph, we have that $\sum_{v \in V(G)} \pi_{S}(v) \leq \frac{1}{2} \sum_{v \in V(G)} \gamma(v)=\frac{1}{2} \times \frac{\Delta^{2}}{2}-|S|$. So we have that the sum of the degrees in $G^{2}[S]$ is at most $\frac{\Delta^{2}}{2}|S|$, which implies that there is a vertex with degree at most $\left\lfloor\frac{\Delta^{2}}{2}-\right\rfloor$ in $G^{2}[S]$. 
Proof of Theorem 4. This lemma implies that there is an order $v_{1}$, $v_{2}, \ldots, v_{n}$ on the vertices of $G=(V, A)$, such that for every $i \leq n$, the vertex $v_{i}$ has at most $\left.\mid \frac{\Delta^{2}}{2}\right\rfloor$ 2-neighbors $v_{j}$, with $j<i$. Given this order on the vertices, we consider the following algorithm:

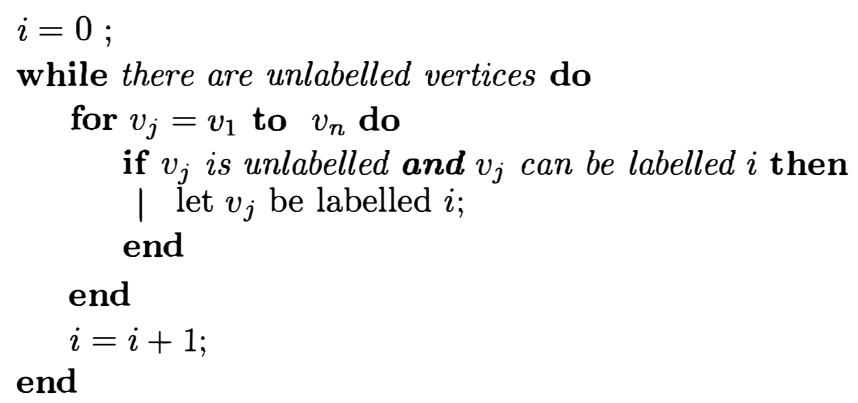

Now consider the last vertex being labelled by this algorithm, say $v$ with label $k$. What could prevent it to be labelled with the value $x<k$, when the algorithm considered the possibility (i.e. when $i=x$ and $v_{j}=v$ )? It is either a neighbor of $v$ that was already labelled with the label $l$, with $x-p<l \leq x$, or a 2-neighbor of $v$ that was labelled $x$. Note that if a 2neighbor of $v$ is labelled $x$ before the possibility was offered to $v$, it implies that this 2-neighbor appears before $v$ in the order. So the 2-neighbors of $v$ posterior to $v$ in the order cannot prohibit a value to $v$. Since $v$ has at most $\Delta$ neighbors and at most $\left\lfloor\frac{\Delta^{2}}{2}-\right\rfloor$ 2-neighbors appearing before $v$ in the order, at most $\left[\frac{\Delta^{2}}{2^{-}}\right\rfloor+p \Delta$ values were refused to $v$. This implies that $k \leq\left\lfloor\frac{\Delta^{2}}{2}\right\rfloor+p \Delta$.

Note that this implies that the algorithm labels the graph in time $O\left(\Delta^{2} n\right)$.

\section{Conclusion}

In this article, we have explored the role of "orientation" in FAP by extending $L(p, q)$-labelling to oriented graphs and introducing oriented $L(p, q)$ labelling. We have computed upper bounds of oriented $L(p, q)$-labelling of trees, bipartite graphs and planar graphs. Note that bounds of $L(p, q)$ labelling of a tree depends on its maximum degree but bounds of oriented 
$L(p, q)$-labelling depends on its structure (see Theorem 1). It indicates that an oriented version of labelling may provide more structrual information of concerned network of FAP than its non-oriented version.

\section{References}

1. K. I. Aardal, S. P. M. V. Hossel, A. M. C. A. Koster, C. Mannino and A. Sassano, Models and Solution Techniques for Frequency Assignment Problems,(available at http://fap.zib.de/survey/)

2. N. Alon and T. H. Marshall, Homomorphisms of edge-coloured graphs and Coxeter groups, J. Algebraic Combin. 8 (1998) 5-13.

3. O. V. Borodin, On acyclic colorings of planar graphs, Discrete Math. 26 (1979), 211-236.

4. O. V. Borodin, A. V. Kostochka, J. Nešetřil, A. Raspaud and E. Sopena, On the maximum average degree and the oriented chromatic number of a graph, Discrete Math. 206 (1999) 77-89.

5. O. V. Borodin, A. V. Kostochka, and D. R. Woodall, Acyclic colourings of planar graphs with large girth, J. London Math. Soc. (2). 60 (1999) 344-352.

6. G. J. Chang, J. Cheng, D. Kuo, Distance-two labelings of digraphs, Tech. Report 2004-011, National Center for Theoritical Sciences at Taipei.

7. G. J. Chang, Wen-Tsai Ke, D. Kuo, D. D. F. Liu and R. K. Yeh, On L(d,1)labelling of graphs, Discrete Math. 220 (2000) 57-66.

8. J. P. Georges and D. W. Mauro, Generalized vertex labelings with a condition at a distance two, Congr. Numer., 109 (1994), 141-159.

9. D. Gonçalves, On the L(d,1)-labelling of graphs, submitted.

10. J. R. Griggs and R. K. Yeh, Labelling graphs with a condition at a distance two, SIAM J. Discrete Math., 5 (1992), 586-595.

11. W. K. Hale, Frequency assignment : Theory and application, Proc. IEEE, 68 (1980), 1497-1514.

12. J. C. M. Janssen, Channel assignment and graph labeling, Handbook of Wireless Networks and Mobile Computing( Editor : Ivan Stojmenvoic), John Wiley and Sons,Inc., Chapter 5, (2002) 95-117.

13. A. V. Kostochka, E. Sopena and X. Zhu, Acyclic and oriented chromatic numbers of graphs, J. Graph Theory 14, No.4 (1997) 331-340.

14. D. Král and R. Škrekovski, A theorem about the channel assignement problem, SIAM J.Discrete Math., 16 (2003), pp. 426-43.

15. B. H. Metzger, Spectrum management technique, Fall 1970, Presentation at 38th National ORSA meeting (Detroit, MI)

16. J. Nešetrril and A. Raspaud, Colored homomorphisms of colored mixed graphs, J. Combin. Theory Ser. B, 80 (2000) 147-155.

17. J. Nešetřil, A. Raspaud and E. Sopena, Colorings and girth of oriented planar graphs, Discrete Math.165, 166, Nos. 1-3 (1997), 519-530.

18. A. Raspaud and E. Sopena, Good and semi-strong colorings of oriented planar graphs, Inform. Process. Lett. 51 (1994) 171-174.

19. E. Sopena, The chromatic number of oriented graphs, J. Graph Theory $\mathbf{2 5}$ (1997) 191-205. 